\title{
Image-guided Tumor Ablation: Standardization of Terminology and Reporting Criteria
}

\section{Citation}

Goldberg, S. Nahum, Clement J. Grassi, John F. Cardella, J. William Charboneau, Gerald D. Dodd, Damian E. Dupuy, Debra Gervais, et al. 2005. "Image-Guided Tumor Ablation: Standardization of Terminology and Reporting Criteria." Radiology 235 (3) (June): 728-739. doi:10.1148/radiol.2353042205.

\section{Published Version}

doi:10.1148/radiol.2353042205

\section{Permanent link}

http://nrs.harvard.edu/urn-3:HUL.InstRepos:33884318

\section{Terms of Use}

This article was downloaded from Harvard University's DASH repository, and is made available under the terms and conditions applicable to Other Posted Material, as set forth at http:// nrs.harvard.edu/urn-3:HUL.InstRepos:dash.current.terms-of-use\#LAA

\section{Share Your Story}

The Harvard community has made this article openly available.

Please share how this access benefits you. Submit a story.

\section{Accessibility}




\title{
Image-guided Tumor Ablation: Standardization of Terminology and Reporting Criteria
}

\author{
S. Nahum Goldberg, MD, Clement J. Grassi, MD, John F. Cardella, MD, J. William \\ Charboneau, MD, Gerald D. Dodd III, MD, Damian E. Dupuy, MD, Debra A. Gervais, MD, \\ Alice R. Gillams, MD, Robert A. Kane, MD, Fred T. Lee Jr, MD, Tito Livraghi, MD, John \\ McGahan, MD, David A. Phillips, MD, Hyunchul Rhim, MD, Stuart G. Silverman, MD, Luigi \\ Solbiati, MD, Thomas J. Vogl, MD, Bradford J. Wood, MD, Suresh Vedantham, MD, and \\ David Sacks, MD for the Society of Interventional Radiology Technology Assessment \\ Committee and the International Working Group on Image-guided Tumor Ablation \\ Department of Radiology, Beth Israel Deaconess Medical Center, Boston, Massachusetts \\ (S.N.G., R.A.K.); Department of Radiology, Lahey Clinic Medical Center, Burlington, \\ Massachusetts (C.J.G.); Department of Radiology, University of Colorado Health Sciences \\ Center, Denver, Colorado (J.F.C.); Mayo Clinic, Rochester, Minnesota (J.W.C.); Department of \\ Radiology, University of Texas Health Science Center, San Antonio, Texas (G.D.D.); Division of \\ Ultrasound, Brown University-Rhode Island Hospital, Providence, Rhode Island (D.D.); \\ Department of Radiology, GI/GU Division, Massachusetts General Hospital, Boston, \\ Massachusetts (D.A.G.); The Middlesex Hospital, London, United Kingdom (A.R.G.); Clinical \\ Science Center, University of Wisconsin, Madison (F.T.L.); Department of Radiology, Ospedale \\ Civile-Vimercate, Milan, Italy (T.L.); U.C. Davis Medical Center, Sacramento, California (J.M.); \\ Department of Radiology, University of Massachusetts Memorial Health Care, Worcester, \\ Massachusetts (D.A.P.); Hanyang University Hospital, Seoul, Korea (H.R.); Department of \\ Radiology, Harvard Medical School, Brigham and Women's Hospital, Boston, Massachusetts \\ (S.G.S.); Department of Radiology, General Hospitals of Busto Arsizio, Busto Arsizio, Italy (L.S.); \\ Institute of Diagnostic/Interventional Radiology, Johann Wolfgang Goethe University Hospital, \\ Frankfurt, Germany (T.J.V.); Radiology Department, NIH Clinical Center, National Institutes of \\ Health, Bethesda, Maryland (B.J.W.); Mallinckrodt Institute of Radiology, WA University School of \\ Medicine, St Louis, Missouri (S.V.); and The Reading Hospital and Medical Center, Reading, \\ Pennsylvania (D.S.)
}

\section{Abstract}

\begin{abstract}
The field of interventional oncology with use of image-guided tumor ablation requires standardization of terminology and reporting criteria to facilitate effective communication of ideas and appropriate comparison between treatments that use different technologies, such as chemical (ethanol or acetic acid) ablation, and thermal therapies, such as radiofrequency (RF), laser, microwave, ultrasound, and cryoablation. This document provides a framework that will hopefully facilitate the clearest communication between investigators and will provide the greatest flexibility in comparison between the many new, exciting, and emerging technologies. An appropriate vehicle for reporting the various aspects of image-guided ablation therapy, including classification of therapies and procedure terms, appropriate descriptors of imaging guidance, and terminology to define imaging and pathologic findings, are outlined. Methods for standardizing the reporting of follow-up findings and complications and other important aspects that require attention when reporting clinical results are addressed. It is the group's intention that adherence to the recommendations will facilitate achievement of the group's main objective: improved precision
\end{abstract}


and communication in this field that lead to more accurate comparison of technologies and results and, ultimately, to improved patient outcomes. The intent of this standardization of terminology is to provide an appropriate vehicle for reporting the various aspects of image-guided ablation therapy.

Recently, the International Working Group on Image-Guided Tumor Ablation published a document entitled "Image-guided Tumor Ablation: Proposal for Standardization of Terms and Reporting Criteria" (1). The main objective was "improved precision and communication in this field that leads to more accurate comparison of technologies and results and ultimately to improved patient outcomes" (1). It was acknowledged by the members of the working group that the new field of image-guided tumor ablation (a branch of interventional oncology) required standardization of terminology and reporting criteria to facilitate effective communication of ideas and appropriate comparison between treatments that use different technologies. On the basis of this premise, a committee was established to author proposed standards, with the proposal unanimously adopted by the committee and ratified by the International Working Group on Image-Guided Tumor Ablation.

The initial goals of the working group's proposal for standardization fall in line with the new initiative of the Society of Interventional Radiology (SIR), which promotes interventional oncology. Along these lines, a Technology Assessment Committee of the SIR has been charged with reviewing and commenting on the standardization of terminology and reporting criteria. Accordingly, the document has been modified in an attempt to align the contents with prior SIR standards and to address additional issues that have been raised by the Technology Assessment Committee. Additionally, we have attempted to respond to several recommendations of the Food and Drug Administration's Center for Devices and Radiological Health in this version of the document. In essence, this independent review and ratification by the SIR Technology Assessment Committee of the prior report (1) represents a continuation of the collaborative initiative to consolidate and unite all investigators and clinicians practicing interventional oncology by providing a common language to describe therapies and outcomes.

\section{CLASSIFICATION OF THERAPIES}

\section{Image-guided Tumor Ablation}

The term tumor ablation is defined as the direct application of chemical or thermal therapies to a specific focal tumor (or tumors) in an attempt to achieve eradication or substantial tumor destruction (2-6). The term "direct" aims to distinguish these therapies from others that are applied orally or via an intravascular or peripheral venous route. We stress the concept of image guidance in the title of our field to reflect our radiological perspective and to highlight that image guidance is critical to the success of these therapies (2-6). Given that most of these therapies can be performed by using a host of imaging modalities (ie, ultrasonography [US], computed tomography [CT], magnetic resonance [MR] imaging, and fluoroscopy), the more general term image guidance is preferred, unless a particular imaging modality is mandated as part of the technique. However, virtually all available ablation techniques can theoretically be used with more than one modality.

While previously, some authors have referred to these procedures as "minimally invasive" or "percutaneous" therapies, these terms should be used only where appropriate. Minimally invasive therapies refer to all therapeutic procedures that are less invasive than conventional open surgery. All percutaneous procedures are therefore minimally invasive; however, not all minimally invasive therapies are performed or applied percutaneously. Indeed, the term "minimally invasive" is often used by surgeons to refer to procedures performed with 
minilaparotomy or laparoscopy (7). Although less invasive than open surgery, these procedures are clearly more invasive than are percutaneous image-guided tumor ablation procedures. Inclusion of the term "percutaneous" as a prefix to "image-guided tumor ablation" is often too limiting because it does not reflect the fact that tumor ablation procedures can also be performed at laparoscopy, endoscopy, or surgery $(8,9)$.

Individual procedures and therapies have often been given multiple different names by various investigators, which can potentially lead to confusion. Hence, we propose and recommend a unified approach to the terminology regarding these therapies. The primary aim of this classification is to provide simplicity and clarity, most notably by eliminating extraneous detail and many acronyms. The committee acknowledges that some acronyms (such as RF and RFA for RF ablation and HIFU for high intensity focused ultrasound) have gained widespread international acceptance. Nevertheless, niche application acronyms should be avoided.

When discrimination between the ablation of malignant versus nonmalignant tissue is needed, the descriptive term "ablation" should still be used, with the type of ablated tissue stated afterward (eg, acetic acid ablation of renal cell carcinoma or RF ablation of atherosclerotic plaque). In other words, the term "thermal (or laser, microwave, etc) ablation" should be used regardless of what is being ablated.

The methods of tumor ablation most commonly used in current practice are divided into two main categories: (a) chemical ablation and (b) thermal ablation. These categories require further definition and standardization of terminology as outlined below. Other interventional oncologic therapeutic approaches, including the percutaneous delivery of genetic material and radioactive seeds and the transcatheter delivery of chemoembolization agents $(10,11)$, may ultimately require better definition but are beyond the scope of this current position article. Nevertheless, many of the issues discussed concerning reporting criteria may likely be equally appropriate for clinical trials with those therapies.

\section{Chemical Ablation}

These therapies are to be classified on the basis of the universally accepted chemical nomenclature of the agent(s), such as ethanol and acetic acid, that induce coagulation necrosis and cause tumor ablation (12-14). For example, the term "ethanol ablation" should replace PEI (percutaneous ethanol instillation or injection), PAI (percutaneous alcohol instillation), and others $(12,13)$. When results are reported, the route (intravascular, intraarterial, or interstitial), substances injected, delivery vehicle (size and type of needle or catheter), and rate of delivery (rapid or bolus injection or a defined rate of infusion) should be specified in the Materials and Methods section of the manuscript. The term "instillation" for the direct delivery of pharmacologic agents is preferred, given that many pharmaceutical agents can be injected (a process that implies rapid percutaneous delivery) or delivered intravascularly with a catheter.

\section{Thermal Ablation Procedures}

This category includes energy sources that destroy a tumor by using thermal energy, with either heat (eg, RF, laser) or cold (cryoablation) (15-45). For thermal therapies, energy is "applied." The term "irradiation of energy," particularly in regard to microwave ablation, is a misnomer and should therefore be avoided.

\section{Procedure Terms}

We prefer to use the term procedure rather than "operation," as the latter implies open surgery. We consider the term session to be synonymous with procedure. A procedure 
refers to a single intervention episode that consists of one or more ablations performed on one or more tumors. Given that procedures may be repeated, a treatment consists of one or more "procedures" or "sessions"; the term is used to define the completed effort to ablate one or more tumors. Each manuscript should state clearly how many "procedures" or "sessions" were needed and why.

\section{Energy Sources and Applicators}

Although the devices are often referred to as "needles" or other nonspecific terms, they do not always conform to these precise classifications. Hence, the term applicator should be used generally to describe all devices. For precision, RF applicators are electrodes, microwave applicators are antennas, and laser applicators are fibers. On the basis of convention and consensus, cryoprobes are used to freeze tissue during cryoablation. For reporting completeness, a reference describing the appropriate applicator(s) should be cited unless the report describes a new prototype device, in which case an appropriate figure and/ or schematic should be provided.

\section{RF Ablation}

This term applies to coagulation induction from all electromagnetic energy sources with frequencies less than $30 \mathrm{MHz}$, although most currently available devices function in the 375 to $500 \mathrm{kHz}$ range (15). The term "radiofrequency" should be written as a single nonhyphenated word. Most devices currently used are monopolar in that there is a single "active" electrode, with current dissipated at a return grounding pad. Bipolar devices have two "active" electrode applicators, which are usually placed in proximity to achieve contiguous coagulation between the two electrodes (16). Additionally, many electrode modifications are now available, as classified below. The type of device and electrode used clearly influences the extent of ablation. Hence, clarity and standardization of terminology are required.

Multitined expandable electrodes-This standard term refers to a family of electrodes that are currently available from several manufacturers $(8,9,17-20)$. The usual embodiment of this type of device is an array of multiple electrode tines that expand from a single centrally positioned larger needle cannula. Currently, these are referred to as "umbrella electrodes," "multi-tined electrodes," "Christmas tree electrodes," "multiple hooked electrodes," or "arrays," but this has led to confusion. Given the number of electrode types that have recently become available and the fact that several multitined devices are now available with variable deployment lengths, the exact electrode model and diameter of the electrode array used must be specified. Also, if a stepped deployment was performed with a multitined device, this too needs to be explained in detail regarding the length and time of deployment.

Internally cooled electrodes-Some devices have a perfusate (such as saline or water) that flows in internal lumina that does not come in direct contact with patient tissues (2123). These should be referred to as "internally cooled" (single or cluster [not "clustered"]) and not confused with perfusion electrodes, as described below. The term cluster electrode is most appropriate to describe internally cooled electrode devices in which three or more closely spaced $(<1 \mathrm{~cm})$ electrodes are used simultaneously to approximate an electrode with a larger diameter (24). Many investigators refer to these electrodes as "an array," which may not adequately reflect the true underlying mechanism for enhanced energy deposition and ablation.

Perfusion electrodes-Electrodes that have small apertures at the active tip that allow fluids (ie, normal or hypertonic saline [see "Adjuvant therapies" below]) to be infused or 
injected into the tissue before, during, or after the ablation procedure should be referred to as perfusion electrodes $(25,26)$. The term replaces descriptions such as "cool-wet," "wet," or "saline-enhanced" electrodes.

Algorithm of energy deposition-The methods used for applying energy have undergone continuous modification and improvement, which has led to substantial confusion and difficulty when the results of studies performed by different groups of investigators are compared. When reporting results, pulsing techniques and other methods for amplifying energy deposition should be succinctly elaborated in the Materials and Methods section. Whenever possible, a reference for the precise algorithm used (eg, ramped energy deposition (18) or impedance regulated (27)) and the model number of the generator should be cited. Additionally, other parameters, including the use of monopolar or bipolar systems, the amount of energy applied (current [milliamperes] or power [watts]), and the duration of ablation should be provided.

Adjuvant therapies-Increased use of adjuvant therapies, such as concomitant percutaneous instillation of sodium chloride solutions to alter electric and thermal conductivity during ablation, are being reported with many variations in technique $(28,29)$. Hence, specific details of the adjuvant used (ie, drug concentration, route and rate of administration, timing in relation to ablation therapy) must be provided. Whenever possible, a reference for the precise algorithm and the rationale for the selected adjuvant therapy used should be provided.

\section{Laser Ablation}

The term laser ablation should replace terms such as "laser intersitital tumor therapy," "laser coagulation therapy," and "laser interstitial photo-coagulation" (30-34). This term should be used for all types of ablation with light energy. Given multiple laser technologies and application methods, including superficial therapy (contact or noncontact mode) or transcutaneous ablation, the term "interstitial" or "direct" can be reported to clarify that laser energy is applied via fibers directly inserted into the tissue.

In addition to the laser source (eg, Nd:YAG, erbium, holmium) and precise wavelength, additional device characteristics must be specified, including (a) type of laser fiber (flexible or glass dome); (b) modifications to the tip (ie, flexible diffusor tip or scattering dome), with dimensions and materials specified; (c) length of applicator and diameter of the optic fiber; and (d) number of laser applicators used (ie, single vs. multiple applicators). Similar to the reporting requirements for RF ablation, additional details of device modification, such as pulsing algorithms and internal cooling of the applicator, should be provided. The following technical parameters also should be provided: (a) laser power, reported as watts per centimeter of active length of laser applicator; $(b)$ total duration of energy application; (c) total amount of energy applied per tumor (mean and range); and (d) sequential or simultaneous energy application to multiple fibers. For energy applied, in addition to the energy measured before the laser enters the fiber, ideally the actual energy output of the fiber or dome prior to the ablation and/or at the end of the procedure should be measured.

\section{Microwave Ablation}

This term should be used for all electromagnetic methods of inducing tumor destruction by using devices with frequencies from $30 \mathrm{MHz}$ to $30 \mathrm{GHz}$ (35-37). The term "microwave ablation" should replace the less succinct terms "percutaneous microwave coagulation therapy" and "microwave coagulation therapy." Additionally, the precise frequency of the device and the type of applicator(s) should be provided. 


\section{Ultrasound Ablation}

There are currently two methods for the application of ultrasound energy: extracorporeal (or transcutaneous) (38) and direct for percutaneous application with a needle-like applicator (39) and for intracavitary (and intracardiac) devices. Hence, the additional nomenclature of "extracorporeal" or "direct" is required prior to focused ultrasound ablation. The term "high intensity" (as commonly found in "high-intensity focused ultra-sound") is not essential because it is vague, imprecise, and implied by the proposed terminology.

\section{Cryoablation}

This term should be used to describe all methods of destroying tissue by means of the application of low-temperature freezing $(40-45)$. The term "cryotherapy" is a suitable alternative because it has been used for many years to describe these methods, and it may also be useful when a literature search on this subject is conducted (44). The phrase "cryo" as a freestanding term is to be avoided because "cryo" is a prefix and not a word. The archaic term "cryosurgery" is also to be avoided as imprecise, given the introduction of newer applicators that can be introduced percutaneously in a minimally invasive fashion.

The freezing of tissue with rapid thawing leads to the disruption of cellular membranes and induces cell death (45). In the past, liquid nitrogen was placed directly on tissue, but with the exception of dermatologic applications, this method is no longer used. In the neck, chest, abdomen, pelvis, and extremities, cryoablation is performed by using a closed cryoprobe that is placed on or inside a tumor. In the two main types of systems, argon gas and either gas or liquid nitrogen are used. Temperatures are measured either at the tip of the cryoprobe or in the handle. In the past, temperature readings from cryoprobes have been a source of controversy because some devices of manufacturers measure the temperature of the coolant as it enters the distal probe tip and others measure at the probe tip itself. Hence, the temperatures at which cryoablation is performed should be specified. For publication purposes, the type of cryoablation system, the gases used, probe dimensions, and length and number of freeze-thaw cycles (active or passive thawing) should also be specified.

\section{Terminology for Describing the Effects of Blood Flow}

All of the thermal methods are negatively influenced by blood flow because it can potentially remove heat before complete tumor ablation is achieved (1-6). (This is also true in reverse for cryoablation, where the premature warming of tissue by blood can limit the effects of freezing on tissue.) The term heat sink effect refers to cooling by adjacent visible (>1-mm-diameter) blood vessels when ablated tissues are heated (46-48). In effect, the shape of the thermal zone of ablation is altered away from the blood vessel, and the overall ablation size is diminished due to removal of heat by flowing blood $(46,47)$, or in the case of cryoablation, due to addition of heat. Although these phenomena serve to protect blood vessels and prevent bleeding from large vessels, they are also a major source of incomplete tumor ablation in many studies involving both thermal and cryoablation. Perfusionmediated tissue cooling (or heating) is a more encompassing term that refers to both the effects of the larger heat sink vessels and the substantial effects of capillary level microperfusion (48). Several strategies have been developed to overcome this problem: pharmacologically decreased blood flow (49), temporary vascular balloon occlusion of a specific vessel during ablation (ie, hepatic artery, hepatic vein, and/or portal vein during intrahepatic ablation) (50), intraarterial embolization and chemoembolization $(36,51,52)$, and a Pringle maneuver (ie, temporary hepatic arterial and portal venous occlusion by means of direct compression of the vessels) during RF ablation at laparotomy $(9,47)$. 


\section{IMAGE GUIDANCE}

While all procedures mentioned in this article refer to tumor ablations guided by imaging, it is important to understand what is meant by the term "image guidance." First, "guidance" refers to procedures in which imaging techniques (eg, fluoroscopy, US, CT, and MR imaging) are used during the procedure. Imaging is used in five separate and distinct ways: planning, targeting, monitoring, controlling, and assessing treatment response (53). Treatments are planned before the procedure, and the assessment of treatment response occurs after the procedure is completed. Targeting, monitoring, and controlling are all performed during the procedure.

\section{Planning}

Imaging techniques, including US, CT, MR imaging, and more recently positron emission tomography (PET), are used to help determine whether patients are suitable candidates for these procedures. Imaging aspects that are particularly important include tumor size and shape, number, and location within the organ relative to blood vessels, as well as critical structures that might be at risk for injury during an ablative procedure. Modalities such as combined PET and CT and three-dimensional reconstructions of cross-sectional imaging data may be used more often in the planning of image-guided tumor ablations in the future.

\section{Targeting}

This term is used to describe the step during an ablation procedure that involves placement of an applicator (eg, an RF electrode or cryoprobe) into the tumor. While much of the current image-guided tumor ablation literature describes the use of techniques such as US and CTto target tumors for purposes of ablating them, targeting is only one aspect of intraprocedural image guidance. Ideal qualities of a targeting technique include clear delineation of the tumor(s) and the surrounding anatomy, coupled with real-time imaging and multiplanar and interactive capabilities. For example, US (54) and some MR imaging $(55,56)$ systems have all of these qualities.

\section{Monitoring}

Monitoring is the term that is used to describe the process with which therapy effects are viewed during a procedure. Changes in imaging that occur during a procedure can and should be used to determine treatment effects. Important aspects of monitoring include how well the tumor and/or target is being covered (ie, included and/or encompassed) by the ablation zone and whether any adjacent normal structures are being affected at the same time. Not all image-guided techniques provide the same degree and types of monitoring. For example, MR imaging is currently the only modality with well-validated techniques for realtime temperature monitoring (40,57-59). The term "monitoring" should not be used to describe response to treatment; for this, "treatment assessment" or "follow-up" is used.

\section{Controlling}

This term is used to describe the intraprocedural tools and techniques that are used to control the treatment. To control an image-guided ablation procedure, the treatment should be monitorable, such that the operator can utilize the image-based information obtained during monitoring to control it. This may simply be repositioning of a therapy applicator on the basis of physician experience, imaging findings, and thermal feedback, or it could be as sophisticated as an automated system that automatically terminates the ablation at a critical point in the procedure (60). 


\section{Assessment of Treatment Response}

Imaging used to assess an image-guided tumor ablation procedure occurs after the procedure is completed and is discussed below as postprocedural imaging (2-6).

\section{PATHOLOGIC AND IMAGING FINDINGS}

The difference between pathologic findings and imaging findings must be stressed by the appropriate selection of terminology. Although in many cases there is a good correlation or overlap between radiological and pathologic findings, this is not invariably the case since over- and under-reporting of the true extent of disease has occurred $(61,62)$. The classic example of this is the assumption that imaging findings (ie, the zone of abnormality on the image) are equivalent to the pathologic findings (ie, the true zone of tumor destruction and/ or treatment effect), which may not always be the case. Hence, careful differentiation between imaging and pathologic findings must be made. This distinction is critical, given that the accuracy of assessment of the extent of tumor destruction by means of imaging findings is limited by the resolution of images and the uncertainty about the viability of cells at the radiographic margins of the zone of ablation.

\section{Zone of Cell Death at Pathologic Examination}

This should be referred to as coagulation or coagulation necrosis. Given that many tumors undergo central necrosis without ablation therapy, the term "coagulation" is preferred over the use of "necrosis" alone because it denotes that the ablation intervention is actively leading to tumor destruction. The more generalized term "coagulation" is preferred over the term "coagulative necrosis" because the latter term has a well-defined meaning in the pathology literature, including the absence of visible nuclei within the dead cells. In actuality, the zone of coagulation, while predominantly consisting of coagulative necrosis, often lacks the classic well-defined histologic appearance of coagulative necrosis in the acute postablation period or even within some zones of adequately ablated tissue for many months following ablation $(22,61,63)$. Indeed, in many cases, specialized stains are required to confirm that cellular death has been achieved after thermal ablation (61).

Another important issue is definition of the zone of ablation at gross pathologic examination. Most thermal therapies induce a central "white zone" of coagulation, a pathologic finding that is generally accepted to represent coagulated tissue, surrounded by a variable "red zone" of hyperemia that is most often absent in ex vivo specimens (64). However, there has been controversy in the measurement; hence, comparison of the "true" size of induced zones of ablation is based on the fact that some authors have reported that this more peripheral red zone also represents ablated tissue, and they include it in their measurements. To avoid confusion, both measurements (white zone alone and white plus red zones) should be provided. At a minimum, the zones included in gross pathologic measurements should be specified.

\section{Zone of Ablation at Postprocedural Imaging}

Appropriate terminology must reflect the fact that although we rely on imaging to define the gross extent of induced coagulation, our accuracy is limited by both spatial and contrast resolution to approximately 2 to $3 \mathrm{~mm}$ (depending on the imaging modality) (61). Hence, in truth, postprocedural imaging findings are only a rough guide to the success of ablation therapy, since microscopic foci of residual disease, by definition, cannot be expected to be identified. The term "ablation zone" can be used to describe the radiological region or zone of induced treatment effect (ie, the area of gross tumor destruction visualized at imaging). The term "lesion" is to be avoided, given the potential confusion about the intended 
meaning, since the term "lesion" has been used to refer to both the "ablation zone" and the underlying tumor to be ablated.

There are two types of imaging findings that are identified after an ablation procedure: those related to zones of decreased perfusion and those in which the signal intensity (at MR imaging), echogenicity (at US), or attenuation (at CT) are altered (1-6). Hence, the imaging strategy and the criteria used to define ablation must be specified. For contrast materialenhanced studies, it is important to recognize that in some organ sites, particularly the kidney, minimal contrast enhancement (ie, $<20 \mathrm{HU}$ for CT) seen soon after ablation can be identified in areas that are subsequently proved at pathologic examination to be uniformly dead tissue (65). (This finding is not well understood but may be due to pseudoenhancement, as has recently been described for renal cysts, or to represent true minimal enhancement from leaky capillaries at the treatment margin.) Other imaging findings also require precise definition.

Transient hyperechoic zone-This is the preferred term to describe the transient (up to $30-90 \mathrm{~min}$ ) zone of increased echogenicity seen at US within and surrounding a tumor during and immediately after RF ablation $(66,67)$. Thereafter, treated tumors often develop mixed echogenicity on follow-up scans. This finding is believed to represent microbubbles of water vapor and other cellular products that form as a result of tissue vaporization during active heating and is most often used as a rough guide as to the extent of induced tumor destruction. However, it is not a precise marker, because both under- and overestimation of the true extent of coagulation have been reported. The term "transient hyperechoic zone" should replace imprecise terms such as "ultrasound cloud," "ultrasound storm," "outgassing," and "microbubble vaporization."

Ablative margin-For many disease processes, particularly for tumors in the liver, the ablation of appropriate margins beyond the borders of the tumor is necessary to achieve complete tumor destruction. The term "ablative margin" is proposed to describe the 0.5 to $1.0-\mathrm{cm}$-wide region that should ideally be ablated in these cases (68). This term is preferable to "surgical margin" (because there is no surgery). It is important to stress that this extent of treatment is not always necessary or desired, particularly during attempts to destroy focal tumors in the kidney in patients with a tendency toward the development of multiple tumors (such as those with von HippelLindau disease), where nephron-sparing and more limited ablation are desired to preserve renal function and avoid dialysis (65).

For normally vascular organs such as the kidney and liver, creation of an ablative margin results in zones of low attenuation and absent perfusion that extend into the parenchyma (26). Increased attenuation occurs in low-density tissues such as perinephric fat (for exophytic renal tumors) $(65,69)$ and the lungs, where the term "ground-glass opacity" is used to describe the imaging findings in the treatment zone surrounding and including the ablated lung tumor (70).

Benign periablational enhancement-This finding can be seen at both pathologic examination and contrast-enhanced imaging and typically suggests a benign physiologic response to thermal injury (initially, reactive hyperemia; subsequently, fibrosis and giant cell reaction) (61). Depending on the protocol used for contrast-enhanced imaging (injection rate and scanning delay), this transient finding can be seen immediately after ablation and can last for up to 6 months after ablation. This finding usually manifests as a penumbra, or a thin rim peripheral to the zone of ablation, that can typically measure up to $5 \mathrm{~mm}$ acutely but most often measures 1 to $2 \mathrm{~mm}$. It is a relatively concentric, symmetric, and uniform process with smooth inner margins, and it needs to be differentiated from "irregular peripheral 
enhancement." The finding is most readily appreciated on the arterial phase CT scans, with persistent enhancement that is often seen on delayed MR images.

Irregular peripheral enhancement-This term represents residual tumor that occurs at the treatment margin. In contrast to "benign periablational enhancement," residual unablated tumor often grows in scattered, nodular, or eccentric pattern. This sign indicates incomplete local treatment (ie, residual unablated tumor). As such, if they are not subject to further therapy, these foci tend to continue to grow. Given the delayed enhancement characteristics of many hypovascular tumors, this finding is often best appreciated in a comparison of portal venous or delayed images ( 3 or more minutes after contrast material injection) with baseline images.

Involution of coagulation-The term "involution" should describe the process by which the body eliminates the zone of induced coagulation over time. The term "shrinkage" should be avoided as imprecise. The term "regression" is likewise to be avoided, given that it is commonly used in the medical oncology literature to describe involution of just the tumor itself, rather than the induced coagulation that often involves both the tumor and the surrounding tissues (ie, the ablative margin). It is important to note that no or minimal involution does not imply treatment failure.

Other imaging findings-Many other imaging findings that represent both host reaction to ablation and repair mechanisms will undoubtedly be seen and reported. Such findings include inflammatory stranding in the acute period after ablation and more chronic findings, such as fibrosis, scarring, and architectural distortion. In general, despite the tendency toward creative description, previously standardized radiological nomenclature should be used to describe these findings whenever possible. The number of new terms to describe these processes should be minimized to wherever new descriptive terminology imparts prognostic value (eg, differentiating between benign peri-tumoral enhancement and residual unablated tumor).

\section{Reporting of Tumor and Ablation Sizes}

Appropriate uniform guidelines and standards are needed to report the extent of induced coagulation. In the past, comparisons between technologies have been made somewhat difficult because some authors report the largest diameter of induced coagulation, some report the average diameter, and some report the short-axis diameter. Additionally, coagulation has occasionally been reported as a volume of ablated tissue without any definition of dimensional measurements. Hence, uniform standards of comparison are essential and must be adopted.

Index tumor-This is the preferred term to describe the initially identified tumor prior to ablation. This tumor should not be referred to as a "lesion" because this term could be confused with the zone of induced coagulation or the region of ablation at imaging.

Size classification of tumors-Actual tumor sizes (mean \pm SD and range if applicable) should be reported. Given that appropriate ablation of adequate margins often represents the rate-limiting step for treatment effectiveness, the maximum diameter of the original tumor must be specified. However, many investigators perform analyses of their results on the basis of stratification of tumor sizes. In this regard, there is currently too much ambiguity and variability in the categorization of tumors by size. Investigators have reported upper limits of 2.0, 2.5, 3.0, and $5.0 \mathrm{~cm}$ in diameter for "small tumors" and 5 or $10 \mathrm{~cm}$ for "large tumors." These differences have made the direct comparison of results with different technologies challenging. We, therefore, recommend that if such categorization is 
performed, the tumor size classification should be standardized according to the following scale: small tumors, diameter of $3 \mathrm{~cm}$ or less; intermediate tumors, diameter of 3 to $5 \mathrm{~cm}$; and large tumors, diameter of more than $5 \mathrm{~cm}$. This classification was determined as the most practical because it parallels the current technical capabilities and effectiveness for most image-guided ablation therapies.

\section{Comparing zones of coagulation among different ablation techniques-Often,}

the extent of induced coagulation is reported in experimental studies as a vehicle for comparing different ablation technologies and parameter modifications $(71,72)$. The extent of induced coagulation should include the short-axis diameter, given that this parameter influences the overall extent of necrosis that can be achieved from a single application of energy and is likely to be an important factor that influences technical success in clinical practice. Hence, while additional parameters can certainly be provided and may be potentially useful, at a minimum this should be the standard that is reported to enable honest comparison between techniques. Of course, given that the ablation of a tumor is performed in three dimensions (ie, it is a volumetric problem), ideally, all three-dimensional measurements of the ablation zone and tumor should be provided, and less ideally, both measurements of the cross-sectional area should be provided. If volume is to be used as the only reported parameter, then a rationale must be specified. Average diameters should be accepted only if the tumor or zone of ablation is truly spherical, varying not more than 2 to 3 $\mathrm{mm}$ in cross-sectional diameter. It is further well known that many devices produce irregularly shaped zones of coagulation. Hence, the degree of uniformity or irregularity in the shape of the ablation zone should be specified.

It is important to stress that reliance on minimum and maximum sizes for the zone of ablation may not be useful for predicting clinical technical effectiveness because other technical factors are likely to be equally important. For instance, depending on the orientation of the energy applicator, a $1 \times 2-\mathrm{cm}$ tumor may be adequately treated with a $2 \times$ $3-\mathrm{cm}$ zone of ablation but not with a $3 \times 2-\mathrm{cm}$ zone of ablation. Ablation diameter or volume may also not tell the entire story. Although a $3 \times 3-\mathrm{cm}$ zone of coagulation may completely cover a 2-cm-diameter tumor when it is correctly positioned; if the zone is off the mark, the entire tumor will not be destroyed.

\section{STANDARDIZATION OF FOLLOW-UP}

Currently, definitions of the appropriate length of follow-up and the time points for technical success are not well established. One investigator's long-term follow-up is often another's short-term follow-up. Hence, specific guidelines need to be adhered to that depend on the type of disease treated and the intended goal of the study. Treatment study goals are generally related to one or more of the following four categories, which usually need to be distinguished from each another: (a) technical success, or, was the tumor treated according to the protocol? (b) technique effectiveness, or, was the tumor effectively ablated? (c) morbidity, or, were critical structures and complications avoided? and (d) outcomes, or, was there some improvement in survival, quality of life, or palliation?

\section{Technical Success}

This term simply addresses whether the tumor was treated according to protocol and was covered completely. Tumor coverage can be assessed either during or immediately after the procedure. For example, MR imaging can be performed to monitor thermal injury and to show that the tumor is being covered completely during the procedure. Contrast-enhanced $\mathrm{CT}$ can be performed immediately after ablation. A tumor that is treated according to protocol and covered completely, as determined at the time of the procedure, is "technically successful." The importance of this term is to help investigators separate those patients in 
whom the protocol could not be executed completely, for either technical reasons or reasons related to comorbid disease, from those that were treated according to protocol.

\section{Technique Effectiveness}

Distinction between "technical success" and "technique effectiveness" must be made. Effectiveness can only be demonstrated with appropriate clinical follow-up. "Technique effectiveness" should therefore refer to a prospectively defined time point (ie, immediately after the last course of a defined ablation protocol or at $1 \mathrm{wk}$ or 1 mo after treatment), at which point "complete ablation" of macroscopic tumor as evidenced at imaging follow-up (or another specified end point) was achieved. The number of sessions (ie, the number of interventional procedures) to achieve the specified end point should likewise be defined. Authors are encouraged to report whether or not this complete ablation included an ablative margin.

Comparison of technical success and effectiveness between various ablation protocols has been challenging because many authors have adopted different terminology or guidelines. This problem is further compounded by our ability, and often the clinical need, to ablate a tumor over many sessions and the possibility of ablating growing foci of local tumor progression months after the initial course of therapy. A window of initial therapy for each ablation technique, during which it is reasonably expected that the tumor will be completely ablated, should be defined. For percutaneous thermal ablation, this window should ideally not exceed an upper limit of either one to four procedures or a specified time frame (up to 1$3 \mathrm{mo}$ ), depending on the size, type, and location of the tumor, as well as the rationale for therapy. We have purposefully left definition of this end point as a broad range, given evolving consensus on defining more specific parameters because each disease process may vary. If complete ablation cannot be achieved within these specified parameters, the tumor should be classified as "unsuccessfully treated."

\section{Primary and Secondary Technique Effectiveness Rates}

Given that multiple treatments with image-guided tumor ablation therapy are often given over the course of the disease, primary and secondary technique effectiveness rates should be reported. The primary effectiveness rate is defined as the percentage of tumors that were successfully eradicated following the initial procedure or a defined course of treatment. The secondary or assisted effectiveness rate includes tumors that have undergone successful repeat ablation following identification of local tumor progression. The term retreatment should be reserved for describing ablation of locally progressive tumor in cases where complete ablation was initially thought to have been achieved on the basis of imaging findings that demonstrated "adequate" ablation of the tumor.

The technical success and technique effectiveness rates are very important as we define the limitations of our technologies, ideally in a manner similar to that used in other disciplines (ie, articles about surgical resection typically report a positive margin rate). Nevertheless, for some protocols, the concepts of local technical success and local tumor progression (ie, technique effectiveness) may have limited impact on the most important outcome parameter: patient survival. For example, use of three to four procedures or 1 month as the window of technique effectiveness may be of secondary importance if the patient lives for 5 years because of the treatment or if the tumor is completely eradicated over multiple courses of ablation therapy over many years.

\section{Complete Ablation versus Partial Ablation}

Many reports have surfaced in which different degrees of partial ablation have been reported $(22,30,33,73,74)$. While consensus has been achieved for defining complete and incomplete 
ablation, there has been a rather arbitrary definition of incomplete ablation. For example, some authors have reported nearly complete ablation as representing greater than $90 \%$ induced necrosis, while others have used a threshold of $95 \%$ necrosis of the index tumor. Nevertheless, it is the opinion of the majority of the committee that this kind of classification of partial ablation is not warranted in an overwhelming majority of cases, given that adequate data are lacking to support a difference in outcome between different levels of partial ablation. Furthermore, such percentages are often estimates and may be inaccurate. Hence, at this time, such stratification should be avoided. It is important to stress that the elimination of this type of stratification does not negate the utility or imply the lack of benefit of tumor ablation as a palliative method. However, other end points should be chosen (see below) when reporting these cases on the basis of the rationale of palliation.

\section{Tumor Palliation}

The specified well-defined rationale for palliative therapy, as well as an appropriate method for assessing outcomes, must be provided. For example, if tumor ablation is valid as a vehicle for pain reduction, pre- and postprocedural pain scales should be obtained $(75,76)$. If ablation is performed to reduce symptoms of a syndrome (such as carcinoid or other hormonally active or paraneoplastic tumors (77)), appropriate documentation of laboratory results from blood or urine obtained before and after therapy must be provided, and other symptom end points and grading systems must be specified and used. Needless to say, one cannot "palliate" asymptomatic tumors. Hence, the term debulking should be used to describe a procedure performed with the sole intent of inducing a reduction of tumor burden.

\section{Failure of Therapy}

Causes of treatment failure-The distinction between local incomplete therapy (tumor progression), new foci of disease in the target organ (especially the liver), and distant malignancy should be distinguished whenever possible and reported. Discrimination between "local tumor progression" and new tumor is important for determining the potential utility (ie, local treatment success rate) of a given method in the setting of many potentially confounding causes of the death of a given patient. Additionally, for patients with cirrhosis, the causes of mortality should be differentiated between hepatic disease and others.

Local tumor progression-Many authors have used the term "local recurrence" to describe the appearance over follow-up of foci of untreated disease in tumors that were previously considered to be completely ablated. This is often a misnomer, given the fact that the tumor in essence did not recur but instead was never completely treated. Hence, the process often described is actually "residual unablated tumor." However, in many cases, it is virtually impossible to determine whether there was incompletely treated viable tumor that continued to grow or if a new tumor (or in the case of hepatocellular carcinoma, "daughter" or "satellite" tumors) grew at the original site. Given this reality, local tumor progression is the preferred term over "local recurrence."

\section{Patient Mortality}

Given that the population of patients that is treated most often are those with cancer, substantial patient mortality that is unrelated to the ablation intervention is anticipated, particularly in clinical studies with long-term follow-up. Therefore, the cause of death should be specified as "tumor related" or due to "other causes." For tumor-related death, further subclassification (eg, differentiating death due to hepatic or diffuse metastatic burden), if possible, will often be useful because it can potentially shed further light on the effectiveness of therapy. 


\section{COMPLICATIONS}

The unified standardized SIR grading system should be used as outlined here $(78,79)$. Complications should be reported by using the SIR standard table so that they can be categorized consistently according to severity. The definition of death is self-explanatory and should be reported on a per-patient basis. Any patient death within 30 days of imageguided tumor ablation should be addressed (SIR classification F). The specific cause of death should be reported, with the potential and degree of causality to the ablation procedure clearly specified. Major and minor complications and side effects should be reported on the basis of the number of ablation sessions on a per-session basis. However, ideally, the number of ablations performed should be included because multiple ablations increase the likelihood of complications $(80,81)$.

The definition of major complication is an event that leads to substantial morbidity and disability, increasing the level of care, or results in hospital admission or substantially lengthened hospital stay (SIR classifications C-E). This includes any case in which a blood transfusion or interventional drainage procedure is required. All other complications are considered minor. It is important to stress that several complications, such as pneumothorax or tumor seeding, can be either a major or minor complications, depending on severity. For tumor seeding, this would depend on whether the ectopic tumor focus can be successfully ablated or otherwise treated.

Differentiation between immediate complications (up to $6-24 \mathrm{~h}$ following the procedure), periprocedural complications (within $30 \mathrm{~d}$ ), and delayed complications (more than $30 \mathrm{~d}$ after ablation) is advised. This stratification will give the reader an idea of when specific complications or side effects are most likely to occur and assist in defining when and how to take adequate precautions. Ablation-related complications should include problems encountered within the periprocedural $(30 \mathrm{~d})$ time period that can be related in any way to the procedure, as well as additional complications identified at delayed follow-up imaging that were judged to be highly likely due to the ablation therapy (eg, biliary ductal stricture, tumor seeding along the needle tract). Additionally, it should be specified which complications are being reported on a patient-by-patient basis (such as death) and whether the denominator represents the number of sessions or the number of tumors.

\section{Side Effects}

Side effects are expected undesired consequences of the procedure that, although occurring frequently, rarely if ever result in substantial morbidity. These include pain, the postablation syndrome, asymptomatic pleural effusions, and minimal asymptomatic perihepatic (or renal) fluid or blood collections seen at imaging. Another such side effect would include asymptomatic imaging evidence of minimal thermal damage to adjacent structures without other evidence of negative sequelae (ie, "collateral damage"). An example of this would include when the zone of ablation extends beyond the liver capsule to include small portions of the diaphragm or kidney. These are not true complications because they do not lead to an unexpected increased level of care.

\section{Pain}

Even with appropriate conscious sedation techniques, patients may experience pain during ablation procedures. Additionally, depending on the organ site, many patients may experience grade 1 to 2 pain for several days, occasionally lasting 1 to 2 weeks following an ablation procedure. Last, thermal ablation, particularly RF, is being used with increased frequency as a method for treating refractory metastatic and primary bone tumor pain $(75,76)$. We, therefore, propose adoption of the Common Toxicity Criteria of the National 
Cancer Institute for reporting pain (this document can be downloaded from the Web site ctep.cancer.gov/reporting/ctc.html) (82): grade 0, no pain; grade 1, mild pain that does not interfere with function; grade 2, moderate pain or pain or analgesics that interfere with function but not interfere with activities of daily living; grade 3, severe pain or pain or analgesics that severely interfere with activities of daily living; and grade 4, disabling pain.

\section{Postablation Syndrome}

This syndrome is a transient self-limiting symptom or sign complex of low-grade fever and general malaise $(44,83)$. The duration depends on the volume of necrosis produced and the overall condition of the patient. If small areas are treated, the patient is unlikely to experience postablation syndrome at all. If very large areas of liver tumors are ablated, the syndrome may persist for 2 to 3 weeks. The majority of patients who have this syndrome will experience some malaise for 2 to 7 days depending on the volume of tumor and surrounding tissue ablated and the integrity of the patient's immune system (ie, patients being treated with steroids or those who have small tumors may experience postablation syndrome).

\section{Follow-up and Outcomes}

Outcomes of interest may include local response, systemic response (pain, cancer syndromes, etc), quality of life, or survival. For those studies that deal with the quality of life, some form of objective measurement must be used both before and after treatment. Ideally, previously validated scales or metrics should be used and appropriately referenced.

Imaging follow-up-Currently, despite a reliance on imaging findings to determine the extent of "unablated residual tumor," there is a lack of consensus on a standard follow-up interval regimen for imaging. The most common approach taken by members of the Working Group includes contrast-enhanced CT or MR imaging within 6 weeks of the initial ablation to determine whether additional ablation therapy is required (many centers perform this examination on the day of the initial procedure) and thereafter every 3 to 4 months to determine technique effectiveness. Imaging intervals may also vary depending on the type of underlying tumor and the goals of treatment. At a minimum, the intervals at which followup imaging was performed should be clearly specified.

Although standard imaging criteria for response assessments have been defined for evaluation of other cancer therapies, these criteria focus almost exclusively on tumor size (84). However, given the heavy reliance on morphologic features other than size in the assessment of results of ablation therapy, exclusive reliance on tumor size does not provide a complete imaging assessment of tumor response and may even lead to erroneous conclusions about the effectiveness of the therapy (85). Therefore, in addition to reporting index tumor and the zone of ablation diameters, assessment of tumor enhancement or lack thereof should also be included in the imaging response assessment following ablation therapy.

Length of follow-up-Currently, many, if not most, published studies for most tumor ablation technologies are preliminary and include only a limited number of patients, with longer periods of follow-up. However, ideally, we will need studies in which large numbers of patients are followed up. When survival and disease-free survival are assessed, an appropriate length of follow-up should be selected on the basis of tumor biology and accepted criteria for other therapies for a given tumor type. For example, the surgery literature has required long-term follow-up of more than 5 years for determining the effect of various therapies on survival for colorectal metastases to the liver or hepatomas $(86-88)$. For other tumors, the appropriate length of follow-up may vary and, indeed for more rapidly 
growing tumors, such as those in the lung, the length of follow-up may be shorter. For slowgrowing tumors, such as primary renal cell carcinoma, the length of follow-up may need to be longer. As a general rule, we advocate the rapid establishment of a consensus on acceptable follow-up times for different tumors. Regardless, on the basis of these concerns, we recommend reporting of the actual mean and/or median length of follow-up (with ranges and/or standard deviations, as appropriate) rather than arbitrary classification into short, intermediate, or long.

\section{OTHER IMPORTANT ASPECTS REQUIRING ATTENTION WHEN REPORTING CLINICAL RESULTS}

\section{Technique Parameters to Be Provided for Publication}

It is our belief that many published series do not provide enough technical detail to permit duplication of the investigators' efforts. This problem is compounded by the fact that there are many different types of ablation equipment on the market and in development and these often change. Hence, the specification of the parameters such as duration of energy applied and manufacturer must be provided. Also, the number of treatment sessions for each tumor should be specified. The procedure approach (ie, whether the procedure was performed percutaneously, laparoscopically, or endoscopically) should also be clearly specified. Additional parameters to be provided for publication should include the following: (a) whether the procedure is performed with general anesthesia or conscious sedation (the specifics of anesthetics and medications administered during the procedure and in the recovery phase should be always reported, including agent, dose, and route), (b) the types of imaging guidance (CT, CT fluoroscopy, US, MR imaging), (c) whether the patient was hospitalized, $(d)$ the number of sessions required to initially achieve technical success, and (e) the subsequent rates of other tumors requiring additional ablation therapy. Last, any repositioning of the applicator during the ablation and the procedure for applicator removal (ie, use of fiber enclosure or other closure devices) should be noted.

\section{Other Study Population Data to Be Reported}

The study population should be rigorously described, including inclusion and exclusion criteria and tumor type and size. The degree of proof of disease required for entry into the study (ie, biopsy, imaging, or serologic criteria) should be clearly specified. Pretreatment evaluation also needs to be reported. In addition to an appropriate focus on anatomy (ie, the organ, tumor size, location, and number), the pretreatment evaluation should also include tumor stage (ie, spread elsewhere), patient comorbidities, age, sex, and overall clinical debility, because outcomes such as mortality will depend on these factors. Obviously, a debilitated cachectic patient with widespread metastases will have a worse outcome following liver RF ablation than will an otherwise well patient.

Findings of a recent study (89) have also suggested the potential complementary effects of chemotherapy and radiation therapy on ablation effectiveness. Hence, the administration of either of these therapies to patients enrolled in clinical trials of ablation should be specified. This should be further classified as having received the conventional oncologic therapies previously, around the time of ablation (within $1 \mathrm{mo}$ ) or during the follow-up period. The specific therapy protocol and the duration of therapy in relation to the ablation therapy should also be provided.

\section{Accurate and Complete Delineation of Ablation Procedures}

Substantial confusion and difficulty in comparing results have arisen regarding the success and complication rates because patients may have had one or more tumors treated over multiple procedure sessions. Ideally, all four parameters (numbers of patients, tumors, 
treatment sessions, and ablation procedures) should be reported whenever possible. Additionally, results are often reported for heterogeneous populations of patients for which varied rationales for the procedure (cure vs. palliation) or outcomes (hepatic metastases vs. hepatocellular carcinoma) have been reported. Therefore, stratification of patients into appropriate categories is advised to avoid confusion and best facilitate extraction of clinically meaningful conclusions.

\section{Minimizing Technical Jargon}

Although substantial technical jargon and marketing terminology appear in the peerreviewed medical literature, these should not be used. For example, colloquial phrasing such as "lesioning" and "burning" are to be avoided when describing the application of thermal energy. Another example is the concept of "roll off" to describe the impedance control algorithm of a device of one particular manufacturer; this term should not be used.

\section{Comparison to Other Treatments}

Given that most reports of image-guided therapy have been relatively small case series, a major benefit of uniform reporting standards is the ability to perform meta-analyses of outcomes to compare therapies (90). Clinical research studies should be reported in such a manner that the results can be directly compared to various cancer therapies, including other forms of image-guided ablation, surgery, radiation therapy, and chemotherapy. The coin of the realm in oncology is survival, disease-free survival, and quality of life stratified according to disease stage and patient functional status $(91,92)$. Nevertheless, there are limited data addressing these issues for most diseases treated with image-guided ablation (93). Thus, the committee wishes to stress the need for studies on organ-by-organ and disease-by-disease bases. Randomized, controlled, and blinded studies are considered the standard for pivotal studies and should be performed when possible (94-97). By the same token, the committee acknowledges both the very real obstacles to performing such studies, (patient recruitment, long periods of data collection, expense, multicenter organization, etc) and the benefit of reporting less robust forms of data, including retrospective studies, case series, and case reports $(94,98)$.

\section{Statistical Evaluation}

Regardless of the study type, rigorous statistical evaluation appropriate for the data collected should be presented $(95,97)$. The primary and secondary study end points should be clearly stated. By bearing in mind that the data from individual studies may need to be treated differently, in general survival outcomes should be reported by using life-table (KaplanMeier) analysis. Patients should be randomized, if possible, and results should be reported on the basis of the intention to treat, whether patients were treated as randomized and whether they were treated per protocol (ie, excluding protocol violations). Outcomes may further need to be stratified according to multiple factors (tumor type, grade, and stage; functional status; comorbidities; etc). Appropriate methods for assessment of quality of life should likewise be selected (99).

\section{CONCLUSIONS}

The intent of this proposal for standardization of terminology is to provide an appropriate vehicle for reporting the various aspects of image-guided ablation therapy. Our intent is to provide such a framework to facilitate the clearest communication between investigators and the greatest flexibility in comparison between the many new, exciting, and emerging technologies. Clearly, this is an ongoing process that will require modification as our understanding of these technologies improves, new treatment paradigms emerge, and greater consensus is achieved on standardizing the reporting of currently unresolved issues. Indeed, 
we welcome constructive feedback from the medical community at large in an attempt to further refine this proposal. Nevertheless, we encourage all of our colleagues to adopt the terminology and reporting strategies outlined in this proposal.

\section{Acknowledgments}

The complete list of authors is S. Nahum Goldberg, MD (coordinated the publication of the initial document and served as topic leader during the subsequent revision of the draft), Clement J. Grassi, MD (chair of the SIR Technology Assessment Committee), John F. Cardella, MD, J. William Charboneau, MD, Gerald D. Dodd III, MD, Damian E. Dupuy, MD, Debra Gervais, MD, Alice R. Gillams, MD, Robert A. Kane, MD, Fred T. Lee, Jr, MD, Tito Livraghi, MD, John McGahan, MD, David A. Phillips, MD, Hyunchul Rhim, MD, Stuart G. Silverman, MD, Luigi Solbiati, MD, Thomas J. Vogl, MD, Bradford J. Wood, MD, Suresh Vedantham, MD, and David Sacks, MD (is SIR Standards Division Councilor). Other members of the Technology Assessment Committee who participated in the development of this clinical practice guideline are John Dean Barr, MD, Gary J. Becker, MD, John J. Borsa, MD, Patricia E. Cole, PhD, MD, Ram VSR Chavali, MD, William B. Crenshaw, MD, Michael D. Dake, MD, Chieh-Min Fan, MD, Ziv Haskal, MD, Randall T. Higashida, MD, Thomas B. Kinney, MD, Sanjoy Kundu, MD, Lindsay Machan, MD, Louis G. Martin, MD, Steven F. Millward, MD, Nilesh H. Patel, MD, Dheeraj Rajan, MD, James E. Silberzweig, MD, Curtis W. Bakal, MD, Curtis A. Lewis, MD, and Kenneth S. Rholl, MD. The authors also wish to acknowledge the other members of the International Working Group on Image-Guided Tumor Ablation who ratified the initial proposal.

\section{Abbreviations}

$\begin{array}{ll}\text { RF } & \text { radiofrequency } \\ \text { SIR } & \text { Society of Interventional Radiology }\end{array}$

\section{References}

1. Goldberg SN, Charboneau JW, Dodd GD 3rd, et al. Image-guided tumor ablation: proposal for standardization of terms and reporting criteria. Radiology. 2003; 228:335-345. [PubMed: 12893895]

2. Goldberg SN, Gazelle GS, Mueller PR. Thermal ablation therapy for focal malignancy: a unified approach to underlying principles, techniques, and diagnostic imaging guidance. AJR Am J Roentgenol. 2000; 174:323-331. [PubMed: 10658699]

3. Dodd GD 3rd, Soulen MC, Kane RA, et al. Minimally invasive treatment of malignant hepatic tumors: at the threshold of a major breakthrough. RadioGraphics. 2000; 20:9-27. [PubMed: 10682768]

4. Gazelle GS, Goldberg SN, Solbiati L, Livraghi T. Tumor ablation with radiofrequency energy. Radiology. 2000; 217:633-646. [PubMed: 11110923]

5. Dupuy DE, Goldberg SN. Image-guided radiofrequency tumor ablation: challenges and opportunities_-part II. J Vasc Interv Radiol. 2001; 12:1135-1148. [PubMed: 11585879]

6. Gillams AR. Thermal ablation of liver metastases. Abdom Imaging. 2001; 26:361-368. [PubMed: 11441547]

7. Vierra M. Minimally invasive surgery. Annu Rev Med. 1995; 46:147-158. [PubMed: 7598451]

8. Siperstein A, Garland A, Engle K, et al. Local recurrence after laparoscopic radiofrequency thermal ablation of hepatic tumors. Ann Surg Oncol. 2000; 7:106-113. [PubMed: 10761788]

9. Curley SA, Izzo F, Ellis LM, Nicolas Vauthey J, Vallone P. Radiofrequency ablation of hepatocellular cancer in 110 patients with cirrhosis. Ann Surg. 2000; 232:381-391. [PubMed: 10973388]

10. Camma C, Schepis F, Orlando A, et al. Transarterial chemoembolization for unresectable hepatocellular carcinoma: meta-analysis of randomized controlled trials. Radiology. 2002; 224:47-54. [PubMed: 12091661]

11. Ramsey DE, Kernagis LY, Soulen MC, Geschwind JF. Chemoembolization of hepatocellular arcinoma. J Vasc Interv Radiol. 2002; 13(pt 2):S211-S221. [PubMed: 12354839] 
12. Livraghi T, Giorgio A, Marin G, et al. Hepatocellular carcinoma and cirrhosis in 746 patients: long-term results of percutaneous ethanol injection. Radiology. 1995; 197:101-108. [PubMed: 7568806]

13. Shiina S, Tagawa K, Niwa Y, et al. Percutaneous ethanol injection therapy for hepatocellular carcinoma: results in 146 patients. AJR Am J Roentgenol. 1993; 160:1023-1028. [PubMed: 7682378]

14. Ohnishi K, Yoshioka H, Ito S, Fujiwara K. Prospective randomized controlled trial comparing percutaneous acetic acid injection and percutaneous ethanol injection for small hepatocellular carcinoma. Hepatology. 1998; 27:67-72. [PubMed: 9425919]

15. Goldberg SN, Dupuy DE. Image-guided radiofrequency tumor ablation: challenges and opportunities—part I. J Vasc Interv Radiol. 2001; 12:1021-1032. [PubMed: 11535764]

16. McGahan JP, Gu WZ, Brock JM, Tesluk H, Jones CD. Hepatic ablation using bipolar radiofrequency electrocautery. Acad Radiol. 1996; 3:418-422. [PubMed: 8796695]

17. LeVeen RF. Laser hyperthermia and radiofrequency ablation of hepatic lesions. Semin Interv Radiol. 1997; 14:313-324.

18. Arata MA, Nisenbaum HL, Clark TW, Soulen MC. Percutaneous radiofrequency ablation of liver tumors with the LeVeen probe: is roll-off predictive of response? J Vasc Interv Radiol. 2001; 12:455-458. [PubMed: 11287532]

19. Rossi S, Buscarini E, Garbagnati F, et al. Percutaneous treatment of small hepatic tumors by an expandable RF needle electrode. AJR Am J Roentgenol. 1998; 170:1015-1022. [PubMed: 9530052]

20. Buscarini L, Buscarini E, Di Stasi M, Vallisa D, Quaretti P, Rocca A. Percutaneous radiofrequency ablation of small hepatocellular carcinoma: long-term results. Eur Radiol. 2001; 11:914-921. [PubMed: 11419162]

21. Lorentzen T. A cooled needle electrode for radiofrequency tissue ablation: thermodynamic aspects of improved performance compared with conventional needle design. Acad Radiol. 1996; 3:556563. [PubMed: 8796717]

22. Solbiati L, Livraghi T, Goldberg SN, et al. Percutaneous radio-frequency ablation of hepatic metastases from colorectal cancer: long-term results in 117 patients. Radiology. 2001; 221:159_ 166. [PubMed: 11568334]

23. Vogl TJ, Mack M, Roggan A, et al. Internally cooled power laser for MR-guided interstitial laserinduced thermotherapy of liver lesions: initial clinical results. Radiology. 1998; 209:381-385. [PubMed: 9807562]

24. Goldberg SN, Solbiati L, Hahn PF, et al. Large-volume tissue ablation with radiofrequency by using a clustered, internally cooled electrode technique: laboratory and clinical experience in liver metastases. Radiology. 1998; 209:371-379. [PubMed: 9807561]

25. Miao Y, Ni Y, Yu J, Zhang H, Baert A, Marchal G. An ex vivo study on radiofrequency tissue ablation: increased lesion size by using an "expandablewet" electrode. Eur Radiol. 2001; 11:18411847. [PubMed: 11511912]

26. Kettenbach J, Köstler W, Rücklinger E, et al. Percutaneous saline-enhanced radiofrequency ablation of unresectable liver tumors: initial experience in 26 patients. AJR Am J Roentgenol. 2003; 180:1537-1545. [PubMed: 12760914]

27. Goldberg SN, Stein M, Gazelle GS, Sheiman RG, Kruskal JB, Clouse ME. Percutaneous radiofrequency tissue ablation: optimization of pulsed-RF technique to increase coagulation necrosis. J Vasc Interv Radiol. 1999; 10:907-916. [PubMed: 10435709]

28. Goldberg SN, Ahmed M, Gazelle GS, et al. Radio-frequency thermal ablation with $\mathrm{NaCl}$ solution injection: effect of electrical conductivity on tissue heating and coagulation-phantom and porcine liver study. Radiology. 2001; 219:157-165. [PubMed: 11274551]

29. Ahmed M, Lobo SM, Weinstein J, et al. Improved coagulation with saline solution pretreatment during radiofrequency tumor ablation in a canine model. J Vasc Interv Radiol. 2002; 13:717-724. [PubMed: 12119331]

30. Vogl TJ, Müller P, Mack M, Straub R, Engelmann K, Neuhaus P. Liver metastases: interventional therapeutic techniques and results, state of the art. Eur Radiol. 1999; 9:675-684. [PubMed: 10354884] 
31. Vogl TJ, Eichler K, Straub R, et al. Laser-induced thermotherapy of malignant liver tumors: general principles, equipments, procedure — side effects, complications and results. Eur J Ultrasound. 2001; 13:117-127.

32. Nolsoe CP, Torp-Pedersen S, Burcharth F, et al. Interstitial hyperthermia of colorectal liver metastases with a US-guided Nd-YAG laser with a diffuser tip: a pilot clinical study. Radiology. 1993; 187:333-337. [PubMed: 8475269]

33. Amin Z, Brown S, Lees WR. Liver tumor ablation by interstitial laser photo-coagulation: a review of experimental and clinical studies. Semin Interv Radiol. 1993; 10:88-100.

34. Bown SG, Rogowska AZ, Whitelaw DE, et al. Photodynamic therapy for cancer of the pancreas. Gut. 2002; 50:549-557. [PubMed: 11889078]

35. Shibata T, Iimuro Y, Yamamoto Y, et al. Small hepatocellular carcinoma: comparison of radiofrequency ablation and percutaneous microwave coagulation therapy. Radiology. 2002; 223:331337. [PubMed: 11997534]

36. Seki T, Tamai T, Nakagawa T, et al. Combination therapy with transcatheter arterial chemoembolization and percutaneous microwave coagulation therapy for hepatocellular carcinoma. Cancer. 2000; 89:1245-1251. [PubMed: 11002219]

37. Lu MD, Chen JW, Xie XY, et al. Hepatocellular carcinoma: US-guided percutaneous microwave coagulation therapy. Radiology. 2001; 221:167-172. [PubMed: 11568335]

38. Jolesz FA, Hynynen K. Magnetic resonance image-guided focused ultrasound surgery. Cancer J. 2002; 8(suppl):S100-S112. [PubMed: 12075696]

39. Deardorff DL, Diederich CJ. Ultra-sound applicators with internal water-cooling for high-powered interstitial thermal therapy. IEEE Trans Biomed Eng. 2000; 47:1356-1365. [PubMed: 11059170]

40. Silverman SG, Tuncali K, Adams DF, et al. MR imaging-guided percutaneous cryotherapy of liver tumors: initial experience. Radiology. 2000; 217:657-664. [PubMed: 11110925]

41. Copper IS. Cryogenic surgery: a new method of destruction or extirpation of benign or malignant tissues. N Engl J Med. 1963; 268:743-749. [PubMed: 14022909]

42. Onik G, Kane R, Steele G, et al. Society of Gastrointestinal Radiologists Roscoe E. Miller Award: monitoring hepatic cryosurgery with sonography. AJR Am J Roentgenol. 1986; 147:665-669. [PubMed: 3529893]

43. Lee FT, Mahvi DM, Chosy SG, et al. Hepatic cryosurgery with intraoperative US guidance. Radiology. 1997; 202:624-632. [PubMed: 9051005]

44. Cozzi PJ, Stewart GJ, Morris DL. Thrombocytopenia after hepatic cryotherapy for colorectal metastases: correlates with hepatocellular injury. World J Surg. 1994; 18:774-777. [PubMed: 7975699]

45. Rubinsky B, Lee CY, Bastacky J, Onik GM. The process of freezing and the mechanism of damage during hepatic cryosurgery. Cryobiology. 1990; 27:85-97. [PubMed: 2311412]

46. Lu DS, Raman SS, Vodopich DJ, Wang M, Sayre J, Lassman C. Effect of vessel size on creation of hepatic radiofrequency lesions in pigs: assessment of the "heat sink" effect. AJR Am J Roentgenol. 2002; 178:47-51. [PubMed: 11756085]

47. Patterson EJ, Scudamore CH, Owen DA, et al. Radiofrequency ablation of porcine liver in vivo: effects of blood flow and treatment time on lesion size. Ann Surg. 1998; 227:559-565. [PubMed: 9563546]

48. Goldberg SN, Hahn PF, Tanabe KK, et al. Percutaneous radiofrequency tissue ablation: does perfusion-mediated tissue cooling limit coagulation necrosis? J Vasc Interv Radiol. 1998; 9:101111. [PubMed: 9468403]

49. Lees WR, Gillams AR, Schumillian C, Branda H. Hypotensive anaesthesia improved the effectiveness of radiofrequency ablation in the liver (abstr). Radiology. 2000; 217(P):228. [PubMed: 11012449]

50. de Baere T, Bessoud B, Dromain C, et al. Percutaneous radiofrequency ablation of hepatic tumors during temporary venous occlusion. AJR Am J Roentgenol. 2002; 178:53-59. [PubMed: 11756087]

51. Rossi S, Garbagnati F, Lencioni R, et al. Percutaneous radio-frequency thermal ablation of nonresectable hepatocellular carcinoma after occlusion of tumor blood supply. Radiology. 2000; 217:119-126. [PubMed: 11012432] 
52. Lencioni R, Cioni D, Donati F, Bartolozzi C. Combination of interventional therapies in hepatocellular carcinoma. Hepatogastroenterology. 2001; 48:8-14. [PubMed: 11269005]

53. Jolesz FA, Silverman SG. Interventional magnetic resonance therapy. Semin Interv Radiol. 1995; 12:20-27.

54. Gazelle GS, Haaga JR. Guided percutaneous biopsy of intraabdominal lesions. AJR Am J Roentgenol. 1989; 153:929-935. [PubMed: 2679000]

55. Silverman SG, Collick BD, Figueira MR, et al. Interactive MR-guided biopsy in an openconfiguration MR imaging system. Radiology. 1995; 197:175-181. [PubMed: 7568819]

56. Lewin JS, Petersilge CA, Hatem S, et al. Interactive MR imaging-guided biopsy and aspiration with a modified clinical C-arm system. AJR Am J Roentgenol. 1998; 170:1593-1601. [PubMed: 9609180]

57. Quesson B, de Zwart JA, Moonen CT. Magnetic resonance temperature imaging for guidance of thermotherapy. J Magn Reson Imaging. 2000; 12:525-533. [PubMed: 11042633]

58. Lewin JS, Connell CF, Duerk JL, et al. Interactive MRI-guided radiofrequency interstitial thermal ablation of abdominal tumors: clinical trial for evaluation of safety and feasibility. J Magn Reson Imaging. 1998; 8:40-47. [PubMed: 9500259]

59. Vogl TJ, Müller PK, Hammerrstingl R, et al. Malignant liver tumors treated with MR imagingguided laser-induced thermotherapy: technique and prospective results. Radiology. 1995; 196:257-265. [PubMed: 7540310]

60. Zientara GP, Saiviroonporn P, Morrison PR, et al. MRI monitoring of laser ablation using optical flow. J Magn Reson Imaging. 1998; 8:1306-1318. [PubMed: 9848743]

61. Goldberg SN, Gazelle GS, Compton CC, Mueller PR, Tanabe KK. Treatment of intrahepatic malignancy with radiofrequency ablation: radiologicpathologic correlation. Cancer. 2000; 88:2452-2463. [PubMed: 10861420]

62. Rendon RA, Kachura JR, Sweet JM, et al. The uncertainty of radio frequency treatment of renal cell carcinoma: findings at immediate and delayed nephrectomy. J Urol. 2002; 167:1587-1592. [PubMed: 11912369]

63. Zervas NT, Kuwayama A. Pathological characteristics of experimental thermal lesions: comparison of induction heating and radiofrequency electrocoagulation. J Neurosurg. 1972; 37:418-422. [PubMed: 4560953]

64. Thomsen S. Pathologic analysis of photothermal and photomechanical effects of laser tissue interactions. Photochem Photobiol. 1991; 53:825-835. [PubMed: 1886941]

65. Pavlovich CP, Walther MM, Choyke PL, et al. Percutaneous radio frequency ablation of small renal tumors: initial results. J Urol. 2002; 167:10-15. [PubMed: 11743264]

66. Leyendecker JR, Dodd GD 3rd, Halff GA, et al. Sonographically observed echogenic response during intraoperative radiofrequency ablation of cirrhotic livers: pathologic correlation. AJR Am J Roentgenol. 2002; 178:1147-1151. [PubMed: 11959720]

67. Goldberg SN, Walovitch RC, Straub JA, Shore MT, Gazelle GS. Radio-frequency-induced coagulation necrosis in rabbits: immediate detection at US with a synthetic microsphere contrast agent. Radiology. 1999; 213:438-444. [PubMed: 10551224]

68. Dodd GD 3rd, Frank MS, Aribandi M, Chopra S, Chintapalli KN. Radio-frequency thermal ablation: computer analysis of the size of the thermal injury created by overlapping ablations. AJR Am J Roentgenol. 2001; 177:777-782. [PubMed: 11566672]

69. Gervais DA, McGovern FJ, Wood BJ, Goldberg SN, McDougal WS, Mueller PR. Radio-frequency ablation of renal cell carcinoma: early clinical experience. Radiology. 2000; 217:665-672. [PubMed: 11110926]

70. Dupuy DE, Zagoria RJ, Akerley W, Mayo-Smith WW, Kavanagh PV, Safran H. Percutaneous radiofrequency ablation of malignancies in the lung. AJR Am J Roentgenol. 2000; 174:57-59. [PubMed: 10628454]

71. de Baere T, Denys A, Wood BH, et al. Radiofrequency liver ablation: experimental comparative study of water-cooled versus expandable systems. AJR Am J Roentgenol. 2001; 176:187-192. [PubMed: 11133564] 
72. Pereira PL, Schenk M, Truebenbach J, Brieger J, Kroeber S, Schmidt D. Liver injury after radiofrequency ablation under physiological perfusion in a porcine model (abstr). Radiology. 2002; 225(P):638.

73. Dupuy DE, Mayo-Smith WW, Abbott GF, DiPetrillo T. Clinical applications of radio-frequency tumor ablation in the thorax. RadioGraphics. 2002; 22(special issue):S259-S269. [PubMed: 12376615]

74. Gillams AR, Lees WR. Survival after percutaneous, image-guided, thermal ablation of hepatic metastases from colorectal cancer. Dis Colon Rectum. 2000; 43:656-661. [PubMed: 10826427]

75. Callstrom MR, Charboneau JW, Goetz MP, et al. Painful metastases involving bone: feasibility of percutaneous CT- and US-guided radio-frequency ablation. Radiology. 2002; 224:87-97. [PubMed: 12091666]

76. Rosenthal DI, Hornicek FJ, Wolfe MW, Jennings LC, Gephart MC, Mankin HJ. Changes in the management of osteoid osteoma. J Bone Joint Surg Am. 1998; 80:815-821. [PubMed: 9655099]

77. Berber E, Flesher N, Siperstein AE. Laparoscopic radiofrequency ablation of neuroendocrine liver metastases. World J Surg. 2002; 26:985-990. [PubMed: 12016479]

78. Burke DR, Lewis CA, Cardella JF, et al. Quality improvement guidelines for percutaneous transhepatic cholangiography and biliary drainage. Society of Cardiovascular and Interventional Radiology. J Vasc Interv Radiol. 1997; 8:677-681. [PubMed: 9232588]

79. Lewis CA, Allen TE, Burke DR, et al. Quality improvement guidelines for central venous access. The Standards of Practice Committee of the Society of Cardiovascular \& Interventional Radiology. J Vasc Interv Radiol. 1997; 8:475-479. [PubMed: 9152925]

80. Vogl TJ, Straub R, Eichler K, Woitaschek D, Mack MG. Malignant liver tumors treated with MR imaging-guided laser-induced thermotherapy: experience with complications in 899 patients (2,520 lesions). Radiology. 2002; 225:367-377. [PubMed: 12409568]

81. Livraghi T, Solbiati L, Meloni MF, Gazelle GS, Halpern EF, Goldberg SN. Treatment of focal liver tumors with percutaneous radio-frequency ablation: complications encountered in a multicenter study. Radiology. 2003; 226:441-451. [PubMed: 12563138]

82. National Cancer Institute. [Accessed December 15, 2004] Cancer therapy evaluation program: common toxicity evaluation manual, version 2.0. Jun 1. 1999 Available at: ctep.cancer.gov/reporting/ctc.html

83. Napier, D.; Dodd, G.; Hubbard, L.; Chintapalli, K.; Chopra, S.; Medina, D. Post ablation syndrome following radiofrequency thermal ablation of liver tumors. Presented at the 29th annual meeting of the Society of Gastrointestinal Radiologists; Kauai, Hawaii. March 12, 2000;

84. Therasse P, Arbuck SG, Eisenhauer EA, et al. New guidelines to evaluate the response to treatment in solid tumors. European Organization for Research and Treatment of Cancer, National Cancer Institute of the United States, National Cancer Institute of Canada. J Natl Cancer Inst. 2000; 92:205-216. [PubMed: 10655437]

85. Chopra S, Dodd GD 3rd, Chintapalli KN, Leyendecker JR, Karahan OI, Rhim H. Tumor recurrence after radiofrequency thermal ablation of hepatic tumors: spectrum of findings on dualphase contrast-enhanced CT. AJR Am J Roentgenol. 2001; 177:381-387. [PubMed: 11461868]

86. Steele G, Bleday R, Mayer RJ, Lindblad A, Petrelli N, Weaver D. A prospective evaluation of hepatic resection for colorectal carcinoma metastases to the liver: gastrointestinal tumor study group protocol 6584. J Clin Oncol. 1991; 9:1105-1112. [PubMed: 2045852]

87. Fong Y, Blumgart LH, Cohen AM. Surgical treatment of colorectal metastases to the liver. CA Cancer J Clin. 1995; 45:50-62. [PubMed: 7804899]

88. Molmenti EP, Marsh JW, Dvorchik I, et al. Hepatobiliary malignancies: primary hepatic malignant neoplasms. Surg Clin North Am. 1999; 79:43-57. [PubMed: 10073181]

89. Goldberg SN, Kamel IR, Kruskal JB, et al. Radiofrequency ablation of hepatic tumors: increased tumor destruction with adjuvant liposomal doxorubicin therapy. AJR Am J Roentgenol. 2002; 179:93-101. [PubMed: 12076912]

90. Stangl, DK.; Berry, DA. Meta-analysis in medicine and health policy. New York, NY: Dekker; 2000.

91. Fisher B. Clinical trials for the evaluation of cancer therapy. Cancer. 1984; 54(suppl):2609-2617. [PubMed: 6498752] 
92. Peto R, Pike MC, Armitage P, et al. Design and analysis of randomized clinical trials requiring prolonged observation of each patient. I Introduction and design. Br J Cancer. 1976; 34:585-612. [PubMed: 795448]

93. Bilchik AJ, Faries M. Radiofrequency ablation of hepatic malignancies: inexpensive and minimally invasive but should it replace resection? Ann Surg Oncol. 2003; 10:1002-1004. [PubMed: 14597435]

94. Sacks D, Marinelli DL, Martin LG, Spies JB. General principles for evaluation of new interventional technologies and devices. J Vasc Interv Radiol. 2003; 14(pt 2):S391-S394. [PubMed: 14514854]

95. Begg CB, McNeil BJ. Assessment of radiologic tests: control of bias and other design considerations. Radiology. 1988; 167:565-569. [PubMed: 3357976]

96. Armitage, P.; Berry, G. Statistical methods in medical research. Oxford, England: Blackwell Scientific; 1987.

97. Rothmann M, Li N, Chen G, Chi GY, Temple R, Tsou HH. Design and analysis of non-inferiority mortality trials in oncology. Stat Med. 2003; 22:239-264. [PubMed: 12520560]

98. Grunkemeier GL, Starr A. Alternatives to randomization in surgical studies. J Heart Valve Dis. 1992; 1:142-151. [PubMed: 1341621]

99. Guyatt GH, Feeny DH, Patrick DL. Measuring health-related quality of life. Ann Intern Med. 1993; 118:622-629. [PubMed: 8452328] 